\title{
Impact of Dietary Fibre Intake on Body Composition and Cardiometabolic Disease Risk Markers
}

\section{Abstract}

Background: Diets higher in fibre have been associated with beneficial effects on cardiometabolic disease (CMD) risk markers including obesity, blood pressure and cholesterol levels. However, the relationship between dietary fibre intake and body composition is unclear. Therefore, the objective of the study was to further assess the association between fibre intake, body composition and CMD risk markers.

Materials and Methods: A single-centred cross-sectional study was conducted in 277 healthy adults $(\mathrm{n}=107 \mathrm{men}$ and $\mathrm{n}=170)$ women with a mean age of 41 (SD 16) y and body mass index (BMI) of 23.9 (SD 3.8) kg/m². Total body composition was measured by dual energy x-ray absorptiometry and dietary intake was assessed with a 4-day weighed food diary.CMD risk markers included fasting lipids and glucose quantified using an ILAB 600 clinical chemistry analyser and clinic blood pressure measured using an Omron blood pressure monitor.

Results: Average AOAC fibre intake in the cohort was 23.0 (SD 9) g/day, with higher intakes found in men (25.0 (SD 10.3) g/day) than women (21.9 (SD 7.8) g/day; $P=0.015)$. AOAC fibre intakes were significantly weakly correlated with weight $\left(\mathrm{r}_{\mathrm{s}}=0.142\right)$, percentage body fat $\left(\mathrm{r}_{\mathrm{s}}=0.193\right)$, bone mineral density $\left(\mathrm{r}_{\mathrm{s}}=0.156\right)$ and fat free mass $\left(\mathrm{r}_{\mathrm{s}}=0.257 ; P \leq 0.009\right)$, and inversely correlated with fasting total cholesterol $\left(r_{s}=-0.124\right)$, low-density lipoprotein (LDL)-cholesterol $\left(r_{s}=0.144\right)$ and total to high-density lipoprotein-cholesterol ratio $\left(r_{s}=-0.129 ; P \leq 0.042\right)$. After stratifying data according to quartiles of AOAC fibre intake and adjusting for covariates (including age, sex, BMI, weight, energy expended per day through physical activity and total energy intake per day) total and LDL-cholesterol concentrations were significantly lower in quartiles (Q)3 (21.0-29.5 g/d) and Q4 (30-63.5 g/d) than Q1 (3.0-18.8 g/d) and Q2 (19.3$20.9 \mathrm{~g} / \mathrm{d})$. Systolic blood pressure was also lower in Q4 than Q1 and Q2 $(P<0.05)$. Anthropometric and body composition measures were not found to be different across quartiles of increasing AOAC fibre intake.

Discussion: Findings from this cross-sectional study have revealed daily fibre consumption greater than $21 \mathrm{~g}$ to be associated with lower fasting total and LDL cholesterol, and intakes $\geq 30 \mathrm{~g}$ also associated with lower systolic blood pressure. With only $9 \%$ of UK adults meeting the current recommended intake, raising public awareness of the importance of dietary fibre is an important strategy for CMD prevention.

\section{Conflict of Interest}

There is no conflict of interest. 